\title{
P02-1-6
}

Poster session

\section{Preconditioning LPS ameliorates stroke-induced brain injury via a reduction of brain matrix metalloproteinases}

\author{
Cheng Wei Huang, Alice Y. W. Chang \\ Institute of Physiology, National Cheng Kung University, Tainan, Taiwan
}

Cerebrovascular disease, including ischemic stroke, is the third leading cause of death worldwide. Clinically and experimentally, a transient increase of blood pressure (BP) and severe inflammatory responses predispose poor prognosis after acute ischemic stroke. Previous study suggested that intraperitoneal (i.p.) pretreatment of lipopolysaccharide (LPS, $0.2 \mathrm{mg} / \mathrm{kg}$ ) expressed a neuroprotective effect via preventing brain injury induced by ischemic stroke. Recently, literatures showed that an increase of matrix metalloproteinases (MMPs) expression was found after cerebral ischemia and was responsible of the degradation of brain extracellular matrix, which contributes to maintain the structural and biochemical support to the surrounding cells. In the present study, we hypothesized that MMPs in hemispheric infarct may participate in regulation of brain injury and the increased blood pressure after ischemic stroke. The ischemic stroke-induced a transient $\mathrm{BP}$ elevation and upregulation of mmps mRNA in the caudate putamen $(\mathrm{CPu})$ were observed in $\mathrm{C} 57 \mathrm{BL} / 6 \mathrm{mice}$ which received middle cerebral artery occlusion (MCAO). Pretreatment LPS $(0.2 \mathrm{mg} / \mathrm{kg}$, i.p. $)$ antagonized strokeinduced BP elevation, infract volume and upregulation of the mRNA expression of mmp1a, mmp3 and mmp9 in CPu. In contrast, stroke-induced mmplb and mmp7 mRNA expressions were potentiated by LPS pretreatment. Furthermore, intracerebroventricular injection of Batimastat (BB-94, a broad spectrum MMP inhibitor) antagonized stroke-elicited transient BP elevation and infract volume. Our results suggested that preconditioning LPS mediated a protective response against ischemic stroke-elicited brain injury and transient BP elevation via a reduction of MMPs expression in $\mathrm{CPu}$. 